\title{
The intrinsic nuclear luminosities of a new sample of medium-distance Seyfert galaxies
}

\author{
Hartmut Winkler* \\ Department of Physics, University of Johannesburg \\ E-mail: hwinkler@uj.ac.za
}

\begin{abstract}
The flux variation gradient technique has enabled the determination of the nuclear colours of many well-known AGN by comparing the total galaxy fluxes at different epochs. It is now established that the intrinsic AGN nuclear colours in the optical are relatively constant throughout the class. This means that measured deviations from these colours are an indicator of partial obscuration and the resulting reddening of the nucleus. The degree of nuclear reddening determined in this manner therefore enables the estimation of the light loss due to circumnuclear matter. From this the intrinsic nuclear luminosity may be calculated. The author is engaged in a long-term observational campaign that seeks to secure photometric magnitudes of a large sample of comparatively rarely studied Seyfert galaxies during two epochs spaced apart by a period of typically 20 years. Initial results confirm that most objects varied sufficiently between the two epochs to compute the flux variation gradients, and hence to determine their intrinsic optical luminosity. This paper discusses the analytical processes necessary to maximise the accuracy of the nuclear luminosity determination. This includes the description of more sophisticated procedures to improve the nuclear component's deconvolution and reddening, and the observational tests performed for this purpose.
\end{abstract}

High Energy Astrophysics in Southern Africa - HEASA2018

1-3 August, 2018

Parys, Free State, South Africa

${ }^{*}$ Speaker. 


\section{Introduction}

The innermost parts of a galaxy containing an active galactic nucleus (AGN) can not be resolved by imaging alone. Therefore one of the most effective means of studying the physical structure and processes of an AGN is to determine its spectral energy distribution (SED). A well-defined SED enables one to establish the presence, nature and relative strength of the various components that combine to produce the SED. Typical components identified in the optical part of the SED of AGN include: i) a thermal emission component from the accretion disk, whose shape is a superposition of Planck curves representative of the temperatures in specific parts of the disk; ii) in the case of radio-loud objects, a synchrotron component normally well-fitted by a power law; iii) an emission-line spectrum; and iv) thermal emission from warm dust at significant distance from the black hole. At shorter wavelengths one may see further components, such as x-ray emission from very close to the black hole, or inverse Compton scattering for some of the most energetic photons (see e.g. [1]). At the opposite end of the spectrum one will sometimes detect radio jets.

The characterisation of the optical SED of AGN and apportionment of its constituent parts is complicated by the fact that i) the SED is invariably contaminated by the emission of normal starlight from the host galaxy, and ii) because the photon beams recorded by an observer may have experienced partial obscuration due to material in the line of sight. In addition, the degree of obscuration in general displays a substantial dependence on wavelength. The correction for these effects is as a rule very difficult in practice.

A technique referred to as the flux variation gradient method [2] uses the recognition that the relationship over time between the fluxes measured with two different photometric filters is linear [3]. Furthermore, unobscured AGN nuclei appear to have a common SED shape in the optical, i.e. they share the same photometric colours [4]. This, with the application of an appropriate reddening law, makes it possible to estimate the amount of nuclear obscuration for objects where these nuclear colours are found to indicate redder colours than in the unobscured case (see also [5, 6]).

While the flux variation gradients determined in the papers listed in the previous paragraph were obtained from a large number of photometric data, it is in theory possible to secure these from two sets of photometric readings only, provided that the two points span a sufficiently large range in AGN luminosity.

This paper describes the rationale and some early results of a long-term project to obtain multicolour photometry of a very large number of AGN, at least once during a bright epoch and once during a faint epoch, and to determine the flux variation gradients and associated intrinsic nuclear luminosities. In particular, it highlights several procedures that will be introduced in this study to enable a higher degree of accuracy in characterising the SEDs.

\section{Processes responsible for light attenuation and spectral distortion}

The light beam from the astronomical source of interest is subjected to attenuation (more generally referred to extinction in astronomy) by gas or small particles. The degree of light loss of the original beam $I_{0}$ is a function of the nature and concentration of the intervening material, which is parameterised by $\kappa$ in Eq. 2.1. Note that $\kappa$, and consequently also the recorded beam $I$, are substantially wavelength dependent. 


$$
I(\lambda)=I_{0}(\lambda) \exp (-\kappa(\lambda))
$$

\subsection{Filters and detectors}

The spectral transmission windows of photometric filters and the spectral response functions of the detectors play a critical role in the measurement of the brightness of astronomical sources. In view of variations in the detector technology and related material composition, the specifications of the filters and detectors are far from uniform. Given that the targets of these measurements also include a vast variety of possible spectral shapes, the determined magnitudes require substantial correction procedures to standardise the data to a uniform system [7, 8].

Note that the magnitude determined for an AGN is particularly sensitive to the transmissivity curve of the filters, as the strong emission line spectrum implies that the target spectral distribution is much more irregular than the far smoother normal galaxy or star spectra. Furthermore, the positions of the emission spikes move relative to the instrumental response function with redshift.

\subsection{The nuclear region}

The obscuration of radiation generated in the nuclear region of an AGN due to circumnuclear dust and gas is common in these types of objects. The unified model of AGN indeed ascribes this as the reason why no broad lines are seen in many AGN spectra [9]. Conversely, the presence of strong photon attenuation in some AGN may offer the opportunity to use this to determine the nature and properties of the attenuating material, and thereby better understand the physical mechanisms in the nuclear region.

Note here that since the bulk of the optical emission in an AGN is understood to originate in the accretion disk and surrounding gas clouds, while $\mathrm{x}$-rays and gamma rays are generated nearer to the black hole, or in parts of a jet, there is no reason why the optical light and high energy photons would experience the same degree of attenuation in this region.

\subsection{Interstellar light attenuation in the AGN host galaxy and the Galaxy}

While most of the extinction of the nuclear beam usually takes place in the gas and dust surrounding the nucleus, there are cases where significant light loss also occurs in the outer reaches of the galaxy, particularly when the galaxy is viewed edge-on. There have been suggestions that wavelength dependence of the interstellar extinction may differ in some galaxies compared to what pertains in most parts of the Milky Way, e.g. in IC 4329A [2]. More clarity could be gained regarding this if accurate flux variation gradients could be secured for more edge-on AGN.

Interstellar extinction correction in our Galaxy is now also achieved far more accurately thanks to the detailed extinction maps produced in recent years [10].

\subsection{Earth atmosphere effects}

Light losses in the Earth's atmosphere are dealt with in photometry by correcting the measured brightness through the application of extinction coefficients appropriate for any particular filter. Since the spectrum of an AGN is fundamentally different from that of a typical star, this again can distort the magnitudes measured with specific filters. One way of confirming the need for different 
extinction coefficients for AGN would be to monitor the magnitude difference between the AGN and a nearby star on the frame. A gradual drift of this magnitude difference while the airmass is changing over a few hours would be a clear indicator that the standard extinction coefficients do not apply to AGN (especially as Seyfert galaxies are not expected to undergo substantial variation in the optical over that time period).

\section{Observational programme and early results}

\subsection{The observations}

$U B V$ observations of a large AGN sample are being obtained with the $1.0 \mathrm{~m}$ telescope at the Sutherland station of the South African Astronomical Observatory. The initial set of images were recorded with an RCA CCD over the period 3-9 Dec 1996, as well as with a Tek8 CCD during the observing week 8-14 Oct 1997. The second set of images were collected during the observing runs 14-27 Dec 2016, 14-20 Jun 2017 and 13-18 Sep 2017 using the STE4 CCD.

Integration times were chosen with consideration given to the target brightness, the presence and phase of the moon and the filter used. All three CCDs utilised have a particularly poor sensitivity when the observation uses the U-filter, and exposures in that band were typically much longer.

Images were reduced utilising flatfields obtained through each filter during twilight. Corrections included the removal of the bias residual as well as pixels affected by cosmic rays.

In all runs the images were calibrated through the observation of E-region standard stars from the list of Menzies et al [11]. In some instances images were not taken in photometric conditions, but for all targets presented here there is data from at least one epoch when the sky was clear of cloud. In all these instances there are one or more comparison stars on the frame whose $U B V$ magnitude could therefore be determined to reasonable accuracy.

\subsection{Example of a preliminary flux variation gradients}

An early example of the calculation of the flux variation gradients and the corresponding extinction of the AGN is illustrated in Fig. 1. $U B V$ frames of the ROSAT-identified Seyfert galaxy $\operatorname{RX} 2256+05\left(\operatorname{RA}(2000)=22 \mathrm{~h} 56 \mathrm{~m} 36 \mathrm{~s}, \delta(2000)=+05^{\circ} 25^{\prime} 15^{\prime \prime}\right)$ were secured on 12 Oct 1997 and 18 Sep 2017. The flux calibration was performed using the calibration coefficients of Bessell and Murphy [7].

The plot in Fig. 1 corresponds to flux variation gradients (i.e. curve slopes) of 0.52 for $U$ flux vs. $V$-flux, and 0.67 for $B$-flux vs. $V$-flux. The values of the flux variation gradients for unobscured AGN will be redetermined once the data for all objects has been reduced and a new list of low-extinction AGN has been identified. If however one assumes the values of $\sim 1.1$ previously determined for both these filter combinations [4], this translates to an AGN nuclear extinction (in the $V$-band) $A_{V} \sim 1.7$.

\subsection{Example of an AGN atmospheric extinction curve}

The extinction of various Seyfert galaxies were determined by tracking the brightness. The comparatively bright Seyfert 1 galaxy ESO 141-G55 has a spectrum characterised by strong broad 


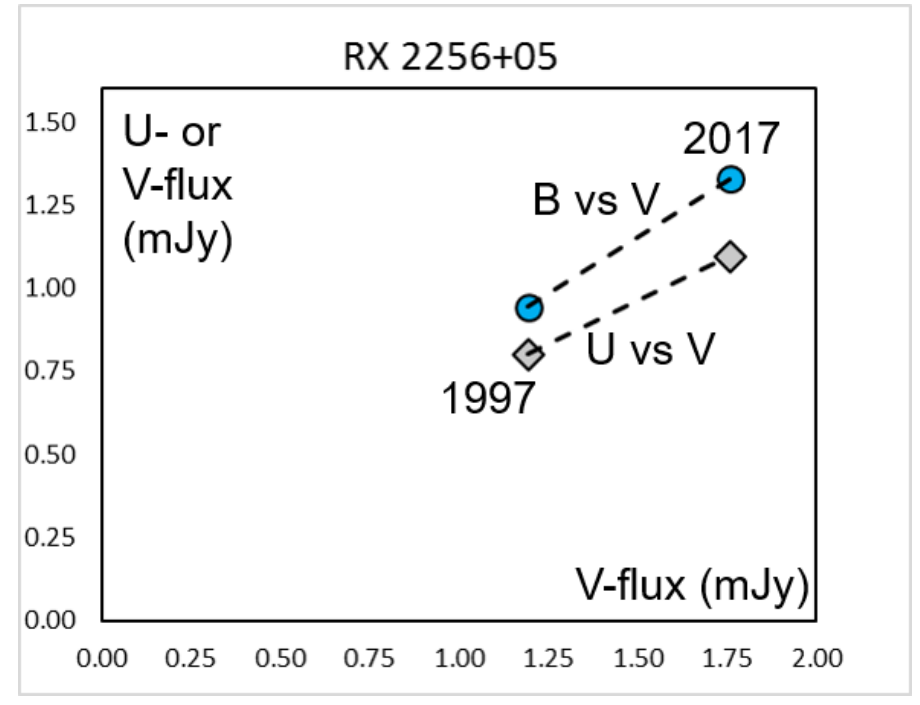

Figure 1: The $B$-flux vs. $V$-flux (blue circles) and $U$-flux vs. $V$-flux (grey diamonds) plots for the observations of RX 2256+05 of 12 Oct 1997 and 18 Sep 2017.

emission lines [12]. This object was observed repeatedly on 18 Sep 2017 in $V, B$ (200 s each) and $U(400 \mathrm{~s})$ filters from when it was close to the meridian until 4.5 hours later. That corresponds to an airmass range of $1.1<\sec \zeta<1.6$ (where $\zeta$ is the zenith angle). The magnitude of the AGN relative to a fairly bright star appearing on the same CCD images is plotted as a function of airmass in Fig. 2. The uncertainties in the comparative magnitudes are typically $0.01 \mathrm{mag}$, i.e. about the size of the symbols used in the Figure.

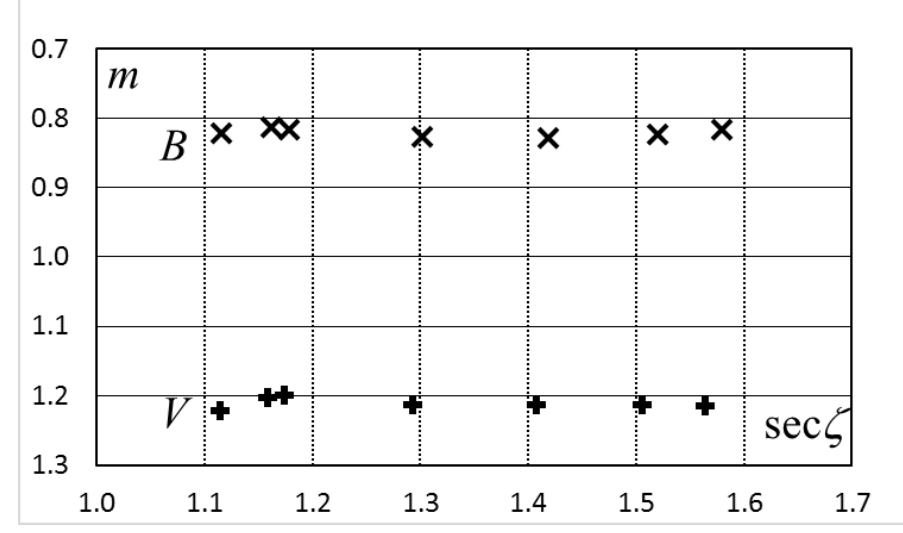

Figure 2: Example of a differential photometry vs. airmass curve. The Seyfert 1 galaxy ESO 141-G55 B and $V$ magnitudes relative to a nearby comparison star as a function of airmass $\sec \zeta$.

Fig. 2 confirms that the extinction coefficients for the $V$ - and $B$-bands applicable to a star are also appropriate for the blue emission-line AGN ESO 141-G55. Further investigations of this nature will illustrate whether other AGN extinction observations will pinpoint the need for different extinction coefficients for other AGN (or other filters). 


\section{Summary}

More sophisticated procedures are being explored that offer the opportunity to determine the extinction of the photon beam from the inner regions of an AGN to greater accuracy. This can reasonably be expected to yield a better definition of the shape of the SED in a large sample of AGN. It will also enable an improved means to separate the host galaxy stellar component from the AGN flux. The initial analysis found that the standard extinction coefficients appear to also be suitable for AGN.

\section{Acknowledgements}

The author thanks the South African Astronomical Observatory for the generous allocation of telescope time.

\section{References}

[1] M. Böttcher, A. Reimer and A. P. Marscher, Implications of the very High Energy Gamma-Ray Detection of the Quasar 3C279, Astrophys. J. 703 (2009) 1168.

[2] H. Winkler, I. S. Glass, F. van Wyk, F. Marang, J. H. S. Jones, D. A. H. Buckley et al., Variability studies of Seyfert galaxies. I - Broad-band optical photometry, Mon. Not. R. astr. Soc. 257 (1992) 659.

[3] J. Choloniewski, The Shape and Variability of the Nonthermal Component of the Optical Spectra of Active Galaxies, Acta Astron. 31 (1981) 293.

[4] H. Winkler, The extinction, flux distribution and luminosity of Seyfert 1 nuclei derived from $U B V(R I)_{C}$ aperture photometry, Mon. Not. R. astr. Soc. 292 (1997) 273.

[5] Y. Sakata, T. Minezaki, Y. Yoshii, Y. Kobayashi, S. Koshida, T. Aoki et al., Long-Term Optical Continuum Color Variability of Nearby Active Galactic Nuclei, Astrophys. J. 711 (2010) 461 [1001.5162].

[6] M. Ramolla, F. Pozo Nuñez, C. Westhues, M. Haas and R. Chini, The stability of the optical flux variation gradient for 3 C 120, Astron. Astrophys. 581 (2015) A93 [1508.01723].

[7] M. Bessell and S. Murphy, Spectrophotometric Libraries, Revised Photonic Passbands, and Zero Points for UBVRI, Hipparcos, and Tycho Photometry, Publ. astr. Soc. Pacific 124 (2012) 140 [1112.2698].

[8] J. Maíz Apellániz, A Recalibration of Optical Photometry: Tycho-2, Strömgren, and Johnson Systems, Astron. J. 131 (2006) 1184 [astro-ph/0510785].

[9] C. M. Urry and P. Padovani, Unified Schemes for Radio-Loud Active Galactic Nuclei, Publ. astr. Soc. Pacific 107 (1995) 803 [astro-ph/ 9506063 ].

[10] E. F. Schlafly and D. P. Finkbeiner, Measuring Reddening with Sloan Digital Sky Survey Stellar Spectra and Recalibrating SFD, Astrophys. J. 737 (2011) 103 [1012 . 4804].

[11] J. W. Menzies, A. W. J. Cousins, R. M. Banfield and J. D. Laing, UBV(RI)c standard stars in the Eand F-regions and in the Magellanic Clouds - a revised catalogue., South African Astronomical Observatory Circular 13 (1989) 1.

[12] H. Winkler, Variability studies of Seyfert galaxies. II - Spectroscopy, Mon. Not. R. astr. Soc. 257 (1992) 677. 